\title{
Contextualising consent
}

No medical ethicist worth their salt would deny that consent is a foundational concept in contemporary medical ethics. Academic ethicists have spent countless hours dissecting consent in extensive theoretical detail: fleshing out its different components, tracing back its moral theoretical heritage, and articulating and defending its boundaries. In medical practice settings, the tendency is to go the other way. Obtaining consent here risks becoming overly proceduralised; a tick box exercise that exists solely to ensure that medical practice is aligned with prevailing professional requirements. In this environment, consent subtly shifts from being used as a noun to being used as a verb. It is common to here doctors and medical students talking about 'consenting' their patients as a distinct and abstracted action that previous research suggests can intrude into a healthcare encounter, depersonalising the delivery of care, and decreasing the quality of communication between doctor and patient ${ }^{1}$.

Writing about the medical research setting, this approach to consent in practice has been described as 'empty ethics', where the activity of obtaining consent from a research participant is stripped out of the real-world context within which its meaning and value can be made sense of. It must be correct that, whilst we can gain much insight from careful philosophical dissections of consent and its value, our ability to shape practice for the better - and to avoid a regimented approach that treats 'consenting' as a mere matter of professional compliance - depends on our ability to take seriously the various contexts in which instances of consent occur. At least in part this is because, stripped bare, consent is about the giving or withholding of a particular type of permission. As they manifest themselves in the world of healthcare, these permissions are embedded in interpersonal interactions, shaped by social context and the vagaries of language. Consent, then, is a messy business. The process of obtaining consent in ethically defensible ways depends on the ability of the practitioner obtaining consent to attend to the particularities of the patient or research participants, the issue under questions, and the wider socio-cultural context in which permissions of all kinds are sought.
It should be immediately apparent from this very brief discussion that obtaining consent from a patient requires a significant degree of skill. The skills to be demonstrated here are not, however, about practitioners' ability to outline the precise formulation of respect for autonomy that consent embodies, or to be able to memorise a list of medical facts that the patients should be told about. Instead, getting consent right requires the making of continual judgements by practitioners about how, what, and when interactions with patients ought to be steered towards clarifying whether a patient agrees with an emergent course of action that is being discussed as part of ongoing clinical care.

This issue of the Journal of Medical Ethics includes a number of papers that, in different ways, aim to navigate the intrinsic messiness of consent such that encounters between practitioners and patients are appropriate for context. One focal point in which the contextualisation of consent manifests itself is in the general relationship between front-line healthcare practice and the legal rules that are put in place to regulate it. Two papers by Farrell and Brazier (see page 85) and by Montgomery and Montgomery (see page $89)$ provide a critical analysis of a recent UK Supreme Court judgement that centres on the informational requirements for valid consent. The position outlined in the Montgomery judgement requires healthcare practitioners to tailor the information they provide to patients by judging what the reasonable patient would expect to know, given the circumstances of the situation, rather than by reference to a professional standard based on medical consensus.

Farrell and Brazier provide a useful and detailed history of the legal heritage of this development, and consider the likely implications for medical law and practice in the UK moving forward. In contrast, Montgomery and Montgomery take issue with the judicial reasoning in the case itself, arguing that a number of misconceptions and problematic inferences were made by the Supreme Court justices who decided the case. One particular point raised by Montgomery and Montgomery is what follows for healthcare expertise in light of the decision by the judges to distinguish between judgements that are purely medical (e.g. whether a particular medication will be effective) from those that involve nonmedical considerations (e.g. which risks to disclose to a patient or what course of treatment to recommend). As they observe, one concern with applying different legal tests to these two kinds of judgements is that it goes against the grain of healthcare practice and training, focused heavily as it is on making holistic judgements about patients' interests and needs within a shared decision-making model.

Whilst much attention on the Montgomery case has focused on the evidence it provides for further advancing the value of respect for patient autonomy in medical practice, this should not be the only conclusion that those working in healthcare take from this judgement or similar legal developments in other jurisdictions. In both education and practice, tailoring information to what a reasonable patient ought to be told, given the individual patient's specific circumstances, is not simply a matter of asking the patient to share her values in order to tailor consent and information disclosure to what matters to the patient. To do so would again be to risk abstracting the consent process from the medical encounter in which it is situated. Knowing how to articulate the patient's values, when to do so, and how to shape a conversation about treatment options in order to enable the patient to make a properly informed decision is a key requirement here, both ethically and legally. The law is just as much concerned with fostering therapeutic partnerships in which doctors, as active partners in decision-making, make correct and qualitative judgements about what treatment options to offer, and what risks to disclose, to their patients; as Farrell and Brazier put it, 'ways of knowing are contextual'. Interpreting these legal developments in this way is far removed from a rudimentary and naïve view that sees the Supreme Court judgement as the legal requirement of doctors to kowtow to patients' articulated values, rather than the evidence-based wisdom of their esteemed colleagues, in order to obtain valid consent.

Changing the scale of analysis, it can also be seen that the challenge of 
obtaining valid consent can be particularly acute in specific practice contexts. Contextualising consent correctly in these settings can be uncertain and open to significant disagreement. In a series of papers, Youngs and Simonds and commentators examine whether information about HIV diagnosis should not be disclosed to a patient when that patient has expressed the desire not to know the outcome of an HIV test. Youngs and Simmonds's criticise the widely held view that the right not to know an HIV test result follows straightforwardly from the value of respecting personal autonomy, instead arguing that the patient's interest in not knowing the test result must be balanced against the foreseeable harms and benefits to the patient and others from this information being disclosed (see pages 95). This position, they believe, can be advanced whilst maintaining the view that the patient retains an absolute right not to undergo the test itself.

Chan (see pages 100) concurs with this view, though is at pains to point out that these harms and benefits need to be calculated carefully. For Andorno (see pages 104) it is the interests of third parties that overrule autonomy-based arguments in favour of the patient's right not to know the outcome of the test result. As he puts it, the more interesting question is not whether a patient has the right not to know but the circumstances in which the patient has a 'duty to know' the test result in order to prevent serious harm to others. Foster (see pages 106) argues against the possibility of differentiating the claim that a patient retains the right to refuse an HIV test, but has no equivalent right not to know the outcomes of that test. Foster's conclusion is essentially that in relation to HIV testing, the context really matters; the correct action to take is likely to be so sensitive to the facts of the case that it makes little sense to draw such a firm dividing line between refusal of undertaking the test and refusal to know the result of the test.

Another context where consent issues can be particularly taxing is in the care of children. When making decisions on behalf of children who are unable to give consent for themselves, Birchley argues against the bioethical zeitgeist in resisting the tendency to replace the best interests standard with a harm threshold for substitute decision-making in this context (see pages 111). Birchley concludes that our time as medical ethicists would be better spent on clarifying the values that ought to inform accurate best interests judgements, rather than seeing the conceptualisation of harm and associated its thresholds as an ethical panacea for determining what is best for children.

Three invited commentators take issue with a number of points raised by Birchley. Nair and Wilkinson (see pages 116) put forward a number of reasons for thinking that the harm threshold is preferable to an approach founded on maximising children's welfare, though convincingly lay out the position that both approaches could operate alongside each other to support the best care of children. Similarly, McDougall (see pages 119) argues that the claims put forward by Birchley would be better focused on the need to develop a comprehensive account of the good for children, and that his empirical criticisms of the harm threshold miss the fact that those who defend this threshold commit themselves to a central normative view of the role of the parents in children's lives that remains unchallenged by Birchley's main thesis. Clearly more work is needed here to make sense of the intersection between an account of the parental role and of children's interests. Again, however, it seems that context is crucial to how such an account ought to be applied. Medical treatment decisions and care placement decisions are decisions that impact on the interests of both parties in very different ways, a point that Foster acknowledges when contrasting medical and family legal frameworks concerning decision-making for children (see pages 123).

Elsewhere in the journal, de Vries reveals how difficulties in contextualising consent emerge due to socio-cultural differences and the unique impacts of novel technologies (see pages 132). The H3Africa Consortium is designed to support projects that apply genomics methodologies to examine diseases that affect African people. Her analysis of consent forms used in H3Africa project reveals very different approaches adopted towards obtaining informed consent from research participants, and dissonances between the researchers' policies on data sharing and incidental findings - both between research studies and the wider H3Africa policy framework. As de Vries acknowledges, precisely why these different approaches have been adopted requires further empirical research. It seems clear, however, that contextualising consent appropriately in this medical research context has some way to go before good research practices are clarified and put into place.

Consent rightly remains an area of medical ethics research that the journal is keen to promote academic dialogue on. Appropriately contextualising consent in different research and practice settings to provide guidance that can help healthcare practitioners and researchers to work through the messy interpersonal interactions within which permissions of this type are formulated, presented and decided upon is a particularly fruitful area for inquiry.

\section{REFERENCES}

1 Taylor K.M., Kelner M. 1987. Informed consent: The physicians' perspective. Social Science and Medicine, 24: 135-43.

2 Corrigan 0. 2003. Empty ethics: The problem with informed consent. Sociology of Health and IIIness, 25: 768-92. 